\title{
Use of enhanced green fluorescent protein to monitor retroviral-mediated gene therapy in human keratinocytes
}

Spandau DF, Marques M, Bierhuizen M, Wagemaker G, Hurwitz S, Pei Y, Breese R, Travers JB. Use of enhanced green fluorescent protein to monitor retroviral-mediated gene therapy in human keratinocytes. Exp Dermatol 2000: 9: 252-257. (C) Munksgaard, 2000

Abstract: Keratinocytes have great promise as targets for gene therapy involving both skin as well as for systemic disorders due to their availability and potential long life span. Improvement of gene transfer into keratinocytes will be greatly facilitated by markers that will allow both rapid detection and efficient selection of transduced cells. For these purposes, a recombinant version of the Aequorea victoria green fluorescent protein that is enhanced for high-level expression in mammalian cells (EGFP) was placed into a replication-deficient retroviral vector. High-titer retrovirus was used to transduce both primary cultures of neonatal foreskinderived human keratinocytes (HK) as well as the immortalized keratinocyte-derived cell line HaCaT. Both cell types stably expressed the EGFP, and this marker allowed rapid purification of transduced cells by fluorescence-activated cell sorting. EGFP expression was seen in HaCaT keratinocytes for at least 40 passages, and the presence of this construct did not effect cell growth, or apoptosis in response to UVB or etoposide. Transduced populations of HK were grafted into SCID mice, resulting in a functional epidermis. EGFP expression was readily seen in vivo by exposing the xenografts to an ultraviolet light source. These studies demonstrate the feasibility of using EGFP as a convenient and rapid marker to monitor keratinocyte gene transfer both in vitro and in vivo.

\author{
D. F. Spandau ${ }^{1,2}$, M. Marques ${ }^{1}$, \\ M. Bierhuizen ${ }^{5}$, \\ G. Wagemaker ${ }^{5}$, S. Hurwitz ${ }^{1}$, \\ Y. Pei $^{1}$, R. Breese ${ }^{3}$ and \\ J. B. Travers ${ }^{1,3,4}$ \\ Departments of ${ }^{1}$ Dermatology, ${ }^{2}$ Biochemistry \\ and Molecular Biology, ${ }^{3}$ Pediatrics, and \\ ${ }^{4}$ Pharmacology, Indiana University School of \\ Medicine Indianapolis, Indiana, USA; ${ }^{5}$ Institute of \\ Hematology, Erasmus University Rotterdam, \\ The Netherlands
}

Key words: keratinocytes - retrovirus - green fluorescent protein - apoptosis

Dan F. Spandau, Department of Dermatology, Indiana University School of Medicine, 975 W. Walnut Street, Rm. 349, Indianapolis, IN 46202-5121 USA

Tel.: 3172747115

Fax: 3172782815

e-mail: DSPANDA@IUPUI.EDU

Accepted for publication 25 October 1999

\section{Introduction}

The human keratinocyte is a very attractive target for gene therapy (reviewed in 1,2). The skin is very accessible, and keratinocytes can be routinely expanded in vitro. Employing the epidermis for gene therapy allows constant and instantaneous monitoring of the treatment site. This feature could permit easy removal of the genetically modified area if needed. These safety concerns as well as the long-lived nature of keratinocytes in vivo have all served to generate interest in gene therapy targeting keratinocytes.

Retroviral vectors are powerful gene transfer ve-

\section{Abbreviations:}

E-GFP, enhanced green fluorescent protein; HK, human keratinocyte; UVB, ultraviolet $\mathrm{B}$ radiation. hicles for the transduction of therapeutic genes into somatic cells (reviewed by 3,4 ). The majority of retroviral vectors in use are designed to express 2 genes, the gene of interest as well as a dominant selectable marker. The selectable marker is used to facilitate the selection of retroviral producer clones, titration of virus on indicator cells, as well as to select target cells, which have been successfully transduced. Common selectable markers for retroviruses include genes which encode for enzymes which inactivate otherwise toxic levels of neomycin, hygromycin or puromycin. In addition, enzymes such as beta-galactosidase that are associated with a color change when incubated with appropriate substrate have been used as reporter genes.

Green fluorescent protein from the jellyfish $A e$ quorea victoria have an inherent green biolumin- 
escence that can be excited optically by blue light or by nonradiative energy transfer $(5,6)$. These qualities have allowed the use of enhanced green fluorescent protein (EGFP) as a reporter molecule in the fluorescent localization and trafficking of various proteins, including membrane-associated g-protein receptors (7). GFP proteins have been expressed by retroviruses and are useful in the monitoring of cell transduction (8).

The high transduction efficiency of retroviral vectors makes them attractive vehicles for gene transduction in keratinocytes. Human keratinocytes are readily transduced by retroviruses $(9,10)$. Transduced keratinocytes have been grafted into immunodeficient mice. Though the first studies resulted in engraftment of transduced keratinocytes in vivo for times measured in days to weeks (11, 12), more recent reports have shown long-term engraftment measured in months (13). One potential problem for current xenografting studies is the lack of a marker, which could be used to monitor gene therapy in a non-invasive manner.

The objective of these studies was to evaluate the feasibility of using EGFP as a marker for retroviral-mediated gene transfer into keratinocytes. Using a replication-deficient Moloney leukemia virus vector that encodes EGFP, we report the ability to transduce both primary cultures of human keratinocytes and the keratinocyte-derived immortalized cell line HaCaT (14). Epidermal cells transduced with this retrovirus fluoresced in vitro, as well as in vivo when grafted into SCID mice. Expression of this protein did not effect growth nor result in enhanced susceptibility to apoptosis in response to UVB or etoposide. These findings indicate that EGFP can be used to evaluate retroviralmediated gene transfer in human keratinocytes.

\section{Materials and methods}

\section{Reagents}

Routine chemicals were obtained from Sigma ( $\mathrm{St}$ Louis, MO, USA). Growth media and supplements were purchased from Life Technologies (Gaithersburg, MD, USA) and fetal bovine serum from Intergen (Purchase, NY, USA).

\section{Generation of the MFG-EGFP retroviral producer cells}

A recombinant replication-deficient retroviral vector was constructed by placing the complete coding sequence of EGFP (originally derived from pEGFP-C1 (Clontech Laboratories Inc, Palo Alto, CA, USA); [8]) under transcriptional control of the MoMLV LTR in the MFG retroviral backbone.
Polyclonal cell populations of ecotropic and amphotropic retroviral producer cell lines $(\mathrm{GP}+\mathrm{E} 86$ and $\mathrm{GP}+e n v \mathrm{Am} 12$, respectively) were generated using this MFG-EGFP plasmid as previously described (8). Clones were derived from FACS-selected populations of amphotropic (aMFGEGFP) producer lines displaying the brightest green fluorescence.

\section{Cells}

Primary cultures of keratinocytes and fibroblasts were obtained from foreskin tissue as previously described (15). These cells were used before the third passage. The immortalized human keratinocyte-derived cell line HaCaT (14) was a kind gift from Dr Petra Boukamp (German Cancer Research Center, Heidelberg, Germany) and were cultured as previously reported (16). Proliferation assays used $2 \times 10^{4}$ cells in $1 \mathrm{ml}$ media plated in 24 well plates. Cells were trypsinized and counted (Coulter) on a daily basis. Doubling time was defined as the time necessary for the cell population to increase $100 \%$ while in log phase growth.

\section{Infection of keratinocytes with MFG-EGFP retrovirus}

Both primary cultures of $\mathrm{HK}$ or $\mathrm{HaCaT}$ cells were transduced with MFG-EGFP retrovirus as previously described (17). Briefly, $10 \mathrm{~cm}^{2}$ dishes of cells (60-70\% confluent) were incubated with $10 \mathrm{ml}$ of supernatants from aMFG-EGFP producer lines along with $8 \mu \mathrm{g} / \mathrm{ml}$ polybrene for $16 \mathrm{~h}$, and repeated. Twenty-four $h$ following the second infection, the cells were then visualized by epifluorescent microscopy or subjected to flow cytometry (FACS Scan; Becton Dickinson).

\section{Measurement of apoptosis in HaCaT cells}

$\mathrm{HaCaT}$ or HaCaT-MFG-EGFP cells were plated at $8 \times 10^{5}$ cells in $10 \mathrm{~cm}^{2}$ dishes for $24 \mathrm{~h}$. Following washing, the cells were either treated with etoposide (Sigma) or UVB irradiation as previously described (18). At various times following treatment, $\mathrm{HaCaT}$ cells were harvested and pelleted. Caspase 3 proteolytic activity in cell lysates was measured using a synthetic fluorogenic substrate (DEVDAMC, Alexis Biochemicals, San Diego, CA, USA; [19]). The cells were suspended in lysis buffer (50 mM PIPES pH 7.0, $50 \mathrm{mM} \mathrm{KCl,} 5$ mM EGTA, 2 $\mathrm{mM} \mathrm{MgCl} 2,1 \mathrm{mM}$ DTT) for $30 \mathrm{~min}$ on ice. Following removal of cellular debris by centrifugation, an aliquot of the cell lysate was added to caspase 3 reaction buffer (100 mM HEPES, pH 7.5, 10\% sucrose, $0.1 \%$ CHAPS, $10 \mathrm{mM}$ DTT, $0.1 \mathrm{mg} / \mathrm{ml}$ 


\section{Spandau et al.}

BSA, and $50 \mu \mathrm{M}$ CPP32-AMC substrate) and incubated at $37^{\circ} \mathrm{C}$ for $1 \mathrm{~h}$. Release of the fluorescent AMC moiety was measured using a Hitachi F2000 Spectrophotometer (excitation, $380 \mathrm{~nm}$; emission, $460 \mathrm{~nm}$ ). The fluorescent intensity was converted to pmoles of AMC by comparison to the fluorescent intensity of standards of AMC (7-amino-4methylcoumarin; Molecular Probes, Eugene, OR, USA). The specific activity of caspase 3 in cell lysates was then determined after the total protein concentration of the cell lysates was measured (NanoOrange protein Quantification Reagent, Molecular Probes). Relative activities were determined by comparing specific activities derived from UVB or etoposide-treated cell lysates versus those derived from untreated cell lysates.

\section{Establishment of xenografts}

Dispersed keratinocytes were grafted into NODLtSz/scid/scid (SCID) mice with minor modifications from published protocols (21). Before use in the grafting protocols, a sample of peripheral blood was screened by flow cytometry for T-cells. Animals used in these studies had $<1 \% \mathrm{~T}$ cells. Briefly, silicone grafting domes (Renner GMBH, Germany) were placed on the dorsum of 2-3 month/old mice using 1\% avertin as anesthesia. A suspension of $5 \times 10^{6} \mathrm{HaCaT}$ or HK-MFG-EGFP cells mixed with $2.5 \times 10^{6}$ primary human foreskinderived fibroblasts was placed into the grafting domes. The grafting domes were gently removed after 2 weeks, and white petrolatum placed on the xenografts on a daily basis. Two-three weeks following removal of the grafting domes, the animals were sacrificed by $\mathrm{CO}_{2}$ asphyxiation, the xenograft was excised, and sections were either placed in formalin for hematoxylin and eosin staining, or snap-frozen in liquid nitrogen in OCT media. Frozen blocks of tissue were cut into $6 \mu \mathrm{m}$ sections and fluorescence visualized with a Nikon Optiphot-2 microscope equipped with epifluorescence.

\section{Results \\ Transduction of keratinocytes with the $M F G$ - EGFP retrovirus}

To assess the potential of EGFP as a marker for monitoring retroviral-mediated gene transfer and expression in human keratinocytes, primary cultures of NHKs or the keratinocyte-derived cell line $\mathrm{HaCaT}$ were incubated with supernatants from aMFG-EGFP. The green fluorescence of transduced cells was readily seen by epifluorescent microscopy. Sorting of these epidermal cells on the basis of EGFP expression demonstrated that the trans- duced cells displayed a strong green fluorescent signal, well separated from the EGFP-negative cells. Analysis of viable transduced cells by FACS revealed EGFP expression in greater than $50 \%$ of $\mathrm{HaCaT}$ and NHKs. The longevity of EGFP expression in $\mathrm{HaCaT}$ cells was assessed by subjecting a clone of HaCaT-EGFP cells at passage 6 and also passage 40 to FACS analysis (Fig. 1). Though substantially reduced, the EGFP was present after 40 continuous passages (approximately one year) in the majority of cells. These studies indicate the feasibility of detection and longevity of retroviralmediated gene expression using EGFP.

\section{Effects of EGFP expression on epidermal function in vitro}

Previous studies have demonstrated that transduction with MFG-EGFP does not affect the outgrowth or differentiation potential of immature hematopoietic cells (8). The influence of EGFP expression on the growth and cytotoxicity of transduced and FACS-sorted (>95\% EGFP-positive) versus untransduced $\mathrm{HaCaT}$ keratinocytes was next evaluated. The spontaneous proliferation of these two populations of cells were essentially identical, with doubling times of approximately $18 \mathrm{~h}$ for parental $\mathrm{HaCaT}$ and $17 \mathrm{~h}$ for HaCaT-MFGEGFP. The next studies examined if retroviral incorporation into the cellular genome and resultant EGFP expression can affect cell cytotoxicity. $\mathrm{HaCaT}$ or HaCaT-MFG-EGFP cells were subjected to UVB irradiation $\left(400 \mathrm{~J} / \mathrm{m}^{2}\right)$ or treatment

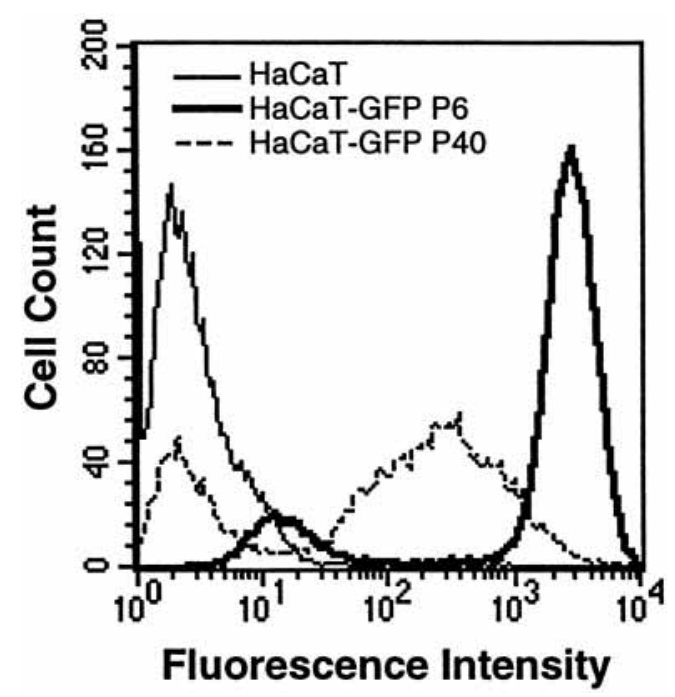

Figure 1. Monitoring E-GFP expression in HaCaT keratinocytes transduced with MFG-EGFP retrovirus. Control HaCaT, or a clone of $\mathrm{HaCaT}$ cells transduced with MFG-EGFP were subjected to flow cytometry at passage 6 , or 40 . 


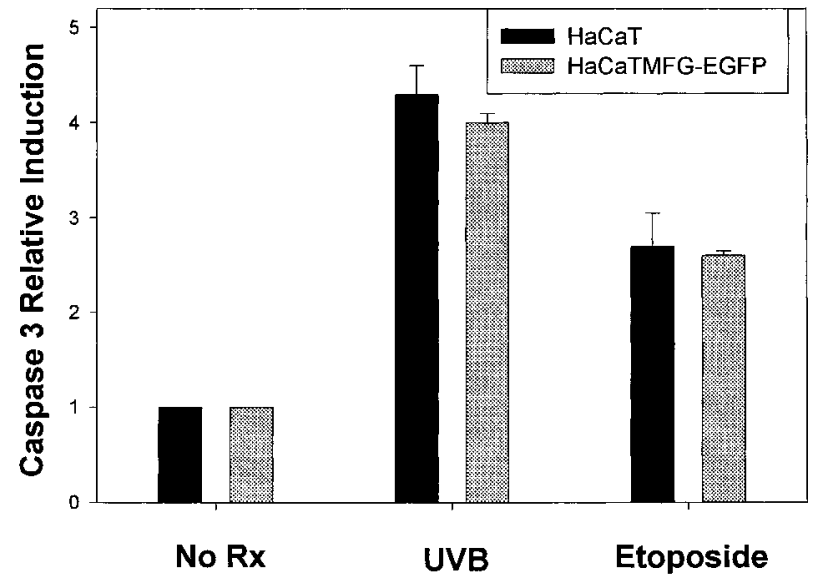

Figure 2. Effect of MFG-EGFP construct on apoptosis induced by UVB or etoposide in $\mathrm{HaCaT}$ keratinocytes. $\mathrm{HaCaT}$ or a population of HaCaTMFG-EGFP cells were treated with 400 $\mathrm{J} / \mathrm{m}^{2} \mathrm{UVB}$ for $6 \mathrm{~h}$ or $5 \mu \mathrm{M}$ etoposide for $24 \mathrm{~h}$. Cells were harvested and caspase 3 enzymatic activity was measured as outlined in Methods. The data depict the mean induction \pm SD of caspase 3 enzymatic activity over untreated cells. The results shown are typical for 3-4 separate experiments using duplicate samples.

with etoposide $(10 \mu \mathrm{M})$, and apoptosis assessed by measurement of caspase 3 activity. We have previously demonstrated that caspase 3 induction in epidermal cells correlates well with other methods for measuring apoptosis (20). As shown in Fig. 2, equal levels of caspase 3 enzymatic activity were noted in parental HaCaT cells versus HaCaTMFG-EGFP following these diverse cytotoxic stimuli. Because the maximum level of caspase 3 induction for $400 \mathrm{~J} / \mathrm{m}^{2} \mathrm{UVB}$ and $10 \mu \mathrm{M}$ etoposide in $\mathrm{HaCaT}$ keratinocytes were 6 and $24 \mathrm{~h}$, respectively, the caspase 3 levels at these two times are depicted in Fig. 2.

Morphologic studies indicated equal numbers of non-viable cells in HaCaT-MFG-EGFP versus parental $\mathrm{HaCaT}$ in response to both UVB and etoposide (data not shown). These studies indicate that retroviral infection with EGFP expression does not have untoward effects on either cellular proliferation, nor does it affect the susceptibility of $\mathrm{HaCaT}$ keratinocytes to programmed cell death.

\section{Establishment of SCID/HK xenografts}

The study of human keratinocyte biology has historically relied upon the in vitro culture of primary HK or carcinoma cell lines. More recently, the increased availability of immunodeficient mice has allowed study of $\mathrm{HK}$ in vivo through xenografts. Several laboratories have demonstrated the ability to create functional xenografts of human keratinocytes genetically manipulated by retroviruses (1113). The following studies were designed to assess whether EGFP is a suitable marker for HK in vivo. Following transduction with EGFP, we grafted populations of primary cultures of HK into SCID mice. As shown in Fig. 3, these cells formed viable xenografts exhibiting a histologically normal epidermis when grafted into SCID mice (Fig. 3D). Green fluorescence was readily visualized in the intact xenografts in vivo by use of an ultraviolet light source (Fig. 3B). Frozen sections of the xenografts from cells transduced with EGFP demonstrated green fluorescence (Fig. 3C). These studies indicate that EGFP is an appropriate and easily visualized marker for retroviral-mediated human keratinocyte gene therapy in vivo.

\section{Discussion}

The accessibility and other features of keratinocytes have made these cells promising targets for gene therapy. Problems in the use of HK in gene therapy include the need for vectors, which will provide long-term stable transduction, as well as the need to direct therapy towards the stem cell population. In addition, the vector used must not disrupt normal cellular function nor result in augmented epidermal damage in response to exogenous stressors such as ultraviolet radiation.

These studies have explored the feasibility of the use of EGFP as a marker for HK both in vitro as well as in vivo. Using a Moloney Leukemia-based recombinant replication-deficient retroviral vector (MFG), we report that $\mathrm{HK}$ and the
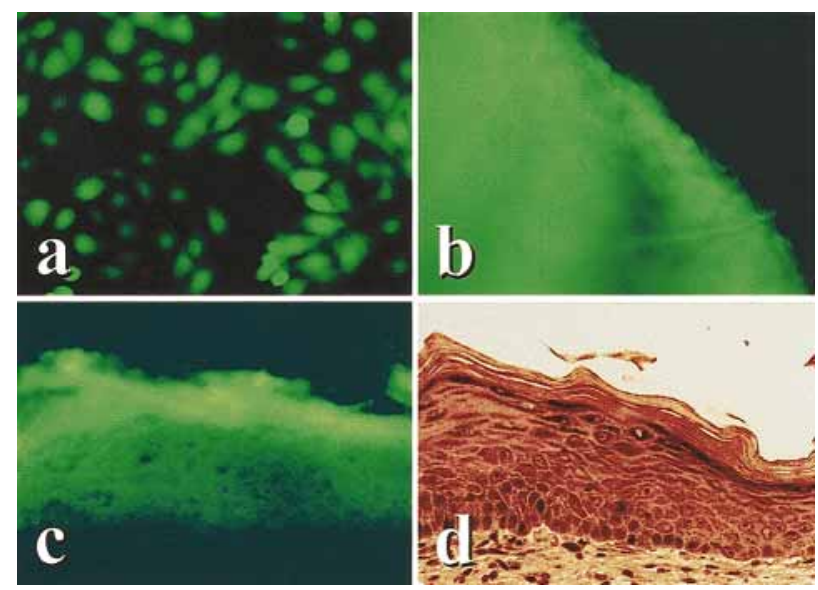

Figure 3. Visualization of human keratinocyte-MFG-EGFP cells. Primary cultures of human keratinocyte were transduced with MFG-EGFP and sorted by flow cytometry and grafted into SCID mice as outlined in Methods. a) Epifluorescence of HKMF-EGFP cells in culture demonstrating green fluorescence. b) Epifluorescence of an intact HKMFG-EGFP/SCID xenograft at 1 month. Sections of the HKMFG-EGFP/SCID xenograft were taken and directly visualized by epifluorescence (c) or stained with hematoxylin and eosin (d). 
keratinocyte-derived epidermal cell line $\mathrm{HaCaT}$ are readily transduced at high efficiencies. Though the EGFP expression decreases over time, the majority of $\mathrm{HaCaT}$ keratinocytes are still positive for this marker after 1 year in continuous culture.

Unlike other selectable markers such as neomycin phosphotransferase, cells expressing EGFP can be rapidly separated from uninfected cells by means of flow cytometry. Thus, in addition to its ability to act as a reporter gene, EGFP can act as a "selectable marker". In contrast to other reporter genes in common use in keratinocytes such as betagalactosidase, EGFP can be easily visualized both in vitro as well as in vivo without compromising the transduced cells. The non-invasiveness of EGFP monitoring would be advantageous, particularly in in vivo gene therapy protocols. The present studies examined short-term (4-6 weeks) expression of GFP-expressing human epidermal xenografts. However, that other laboratories have reported that HK transduced with other retroviral vectors results in stable expression of the transgene in immunodeficient mice (13), coupled with our longterm studies with HaCaTMFG-EGFP cells (Fig. 1) suggest that EGFP-expressing vectors could be used for long-term use in vivo.

It is important that proteins used as markers for gene therapy do not have adverse effects upon the cells, which express them. Growth studies with $\mathrm{HaCaT}$ keratinocytes indicate that the presence of EGFP does not effect spontaneous proliferation in this immortalized cell line. In addition, programmed cell death due to two diverse stimuli was not affected by the presence of the incorporated provirus into the genomic DNA, or expression of the EGFP.

In summary, these studies demonstrate the feasibility of using EGFP as a marker for keratinocyte gene therapy, both in vitro as well as in vivo. This protein seems well tolerated by epidermal cells. Though the present studies use HK xenografts in an immunosuppressed mouse, the recent development of humanized forms of GFP (22) could allow use of this family of proteins for human gene therapy protocols.

\section{Acknowledgements}

The authors wish to thank Dr Antoinette Hood (Departments of Pathology and Dermatology, Indiana University) for her assistance in the preparation and interpretation of histological sections from the xenografts. The authors thank Dr David Williams (Department of Pediatrics and Howard Hughes Medical Institute, Indiana University) for supplying the SCID mice. These studies were supported in part by grants from the Dermatology Foundation, The American Cancer Society Institutional Grant IRG 161-K, The Showalter Memorial Founda- tion, and the National Institutes of Health grants K08AR1993, R01HL62996, and 2P30 DK48218-06. Dr Marques was supported through a scholarship from FAPESP (Brazil).

\section{References}

1. Khavari P A. Gene therapy for genetic skin disease. J Invest Dermatol 1998: 110: 462-467.

2. Taichman L B. Gene therapy. Periodontal Clin Invest 1998: 20: 7-9.

3. Robbins P D, Ghivizzani S C. Viral vectors for gene therapy. Pharmacol Ther 1998; 80: 35-47.

4. Moritz T, Williams D A. Gene transfer into the hematopoeitic system. Curr Opin Hematol 1994: 1: 423-428.

5. Shimomura O, Johnson F H. Chemical nature of bioluminescence system in coelenterates. Proc Natl Acad Sci (USA) 1975: 72: 1546-1549.

6. Prendergast F G. Biophysics of the green fluorescent protein. In: Sullivan K F, Kay S A, eds. Green Fluorescent Proteins. San Diego; Academic Press, 1999; 1-17.

7. Barak L S, Ferguson S S, Zhang J, Martenson C, Meyer T, Caron M G. Internal trafficking and surface mobility of a functionally intact beta-2-adrenergic receptor-green fluorescent protein conjugate. Mol Pharmacol 1997: 51: 177184.

8. Bierhuizen M F, Westerman Y, Visser T P, Dimjati W, Wognm A W, Wagemaker G. Enhanced green fluorescent protein as selectable marker of retroviral-mediated gene transfer in immature hematopoietic bone marrow cells. Blood 1997: 90: 3304-3315.

9. Garlick J A, Katz A B, Fenjves E S, Taichman L B. Retrovirus-mediated transduction of cultured epidermal keratinocytes. J Invest Dermatol 1991: 97: 824-829.

10. Stockschlaeder M A, Storb R, Osborne W R, Miller A D. L-histidinol provides effective selection of retrovirus-vectortransduced keratinocytes without impairing their proliferative potential. Hum Gene Ther 1991: 2: 33-39.

11. Gerrard A J, Hudson D L, Brownlee G G, Watt F M. Towards gene therapy for hemophilia B using primary human keratinocytes. Nat Genet 1993: 3: 180-183.

12. Page S M, Brownlee G G. An ex vivo model for gene therapy of hemophilia B. J Invest Dermatol 1997: 109: 139145.

13. Kolodka T M, Garlick J A, Taichman L B. Evidence for keratinocyte stem cells in vitro: long-term engraftment and persistence of transgene expression from retrovirus-transduced keratinocytes. Proc Natl Acad Science (USA) 1998: 95: 4356-4361.

14. Boukamp P, Petrussevska R T, Breitkreutz D, Hornung J, Markham A, Fusenig N E. Normal keratinization in a spontaneously immortalized aneuploid human keratinocyte cell line. J Cell Biol 1988: 106: 761-778.

15. Spandau D F. Distinct conformations of p53 are observed at different stages of keratinocyte differentiation. Oncogene 1994: 9: 1861-1868.

16. Travers J B, Harrison K A, Johnson C A, Clay K L, Morelli J G, Murphy R C. Platelet-activating factor biosynthesis induced by various stimuli in $\mathrm{HaCaT}$ keratinocytes. J Invest Derm 1996: 107: 88-94.

17. Pei Y, Barber L A, Murphy R C et al. Activation of the epidermal platelet-activating factor receptor results in cytokine and cyclooxygenase-2 biosynthesis. J Immunol 1998: 161: 1954-1961

18. Barber L A, Spandau D F, Rathman S C et al. Expression of the platelet-activating factor receptor results in enhanced ultraviolet $\mathrm{B}$ radiation-induced apoptosis in a human epidermal cell line. J Biol Chem 1998: 273: 18891-18897.

19. Hurwitz S A, Spandau D F. Quantitative analysis of UVB- 
induced apoptosis in human epidermis. Exp Dermatol 2000: 9: 185-191.

20. Nicholson D W, Ali A, Thornberry N A et al. Identification and inhibition of ICE/CED3 protease necessary for mammalian apoptosis. Nature 1995: 376: 37-43.
21. Strickland J E, Dlugosz A A, Hennings H, Yuspa S H. Carcinogenesis 1993: 24: 205-209.

22. Chishima T, Miyagi Y, Wang X et al. Visualization of the metastatic process by green fluorescent protein expression. Anticancer Res 1997: 17: 2377-2384. 